\title{
Sloan Letter Eye Chart 100 Percent
}

National Cancer Institute

\section{Source}

National Cancer Institute. Sloan Letter Eye Chart 100 Percent. NCI Thesaurus. Code C158246.

A diagrammatic method of vision testing that utilizes black letters on a white background to assess high contrast visual acuity that uses the Sloan optotypes and a 100\% contrast level. 